\title{
The Influence of Microwave Heating on Dyeing Time and Dyeing Rate in Cotton Fabrics
}

\author{
Huang De-chao
}

(Dongguan Polytechnic, Guangdong, Dongguan, 523808)

Keywords: Microwave heating; Cotton fabric; Dyeing rate; Dyeing time; Impact

\begin{abstract}
Relevant researches show that microwave heating caused certain effect on reactive dyes' dyeing rate. In order to verify the above viewpoint, this paper designed the following experiments: study microwave solid color dyeing, microwave fixation conventional dyeing, dyeing conventional microwave fixation with conventional dyeing process as reference, in order to investigate microwave heating' $s$ influence on dyeing rate and dyeing time in cotton fabrics. Experimental results show that compared with conventional dyeing fixation, microwave dyed solid color will reduce fixation time, and increase per unit time of reactive dyes dyeing rate on cotton fabric. The integrated use of conventional technology and microwave technology can reduce dyeing time or fixation time as well as ensure dyeing rate.

With the development of science and technology, microwave components improve the performance and at the same time constantly reduce cost, which lays a solid foundation for the wide range of application and popularization of microwave, and greatly facilitates life and production in China. In dyeing industry, the earliest application of microwave was in textile material drying, and as dyeing technology and development of microwave technology, it was applied to dyeing and fixation. Rapid heating with microwave changes additives molecular orientation, and makes it speed up the movement and increase friction, and then achieves the goal of rapid dyeing fixation.
\end{abstract}

\section{Test}

\section{A. Material}

Reagents: anhydrous sodium carbonate (analysis); Reactive turquoise blue KN - G (industrial); Pure, anhydrous sodium sulfate (analysis); Active orange K - R (industrial) [1].

Fabric: cotton bleached fabric.

\section{B.Equipment}

WP microwave oven; 721 spectrophotometer; Y(B)571B color fastness friction tester; AP oscillation small prototype; SW-12 wash fastness tester [2].

\section{C.Dyeing process}

1. Conventional dyeing process prescription and process

(1) Conventional dyeing process prescription: sodium carbonate, sodium sulfate solution, active orange $\mathrm{K}-\mathrm{R}$. Bath ratio $=1: 30$

(2) Conventional dyeing process:

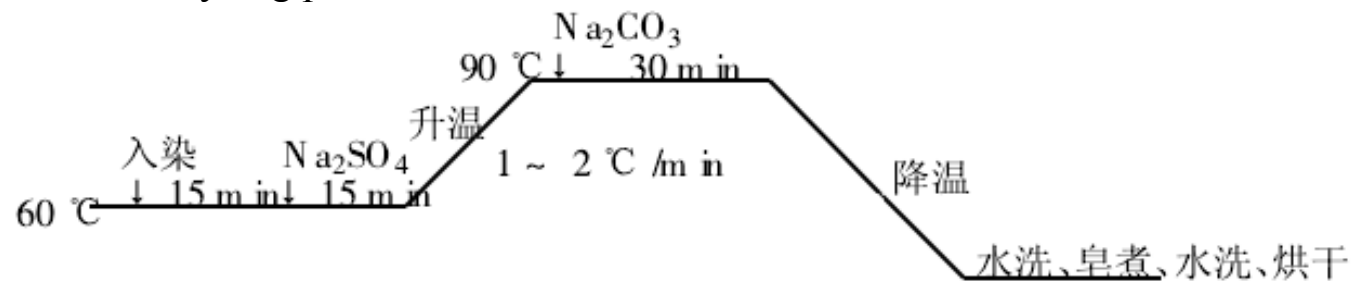

Figure 1 Fixation conventional dyeing process 
2.Microwave dyeing process

(1) Microwave dyeing prescription is the same as conventional dyeing prescription

(2) Microwave dyed solid color process

(a) Configure dyeing in triangle bottle according to dyeing prescription, static to room temperature. (b) Join solid Na2SO4, stir until Na2SO4 fully integrated into dyeing liquid dye solution. (c) Putpure cotton bleaching fabric into it, and then place it in microwave oven for microwave dyeing until the end of dyeing. (4) Join solid Na2CO3once again and place it in microwave oven for microwave solid color.

\section{Dyeing rate test}

\section{Conventional dyeing rate test}

Configure dyeing liquid according to conventional dyeing process prescription, and then put it evenly to A, B, C, D, E, F, G, H triangle bottles. In A triangle bottle, do conventional dyeing, and the time is $5 \mathrm{~min}$, then take it out; B, C, D triangle bottles operation modes are the same, but for 10, $20,30 \mathrm{~min}$, respectively. In E triangle bottle, do regular solid color, and the time is $5 \mathrm{~min}$, then take it out; F, G, H triangle bottles operating modesare the same as E triangle bottle way, but time are 10, 20, $30 \mathrm{~min}$, respectively. Dilute the dyeing liquid residue with distilled water to $200 \mathrm{ml}$, and measure $\mathrm{An}$ in the maximum absorption wavelength in dye absorbance. Dyeing percentage (E) formula is [3] :

$\mathrm{E} / \%=\left[1-\frac{A_{\mathrm{n}}}{A_{0}}\right] \times 100 \%$

In it, A0 is blank dyeing absorbance; An is absorbance of dyeing liquor (absorbance is measured by 721 spectrophotometer).

2.Dyeing rate test in microwave dyeing

Every 1 min,sample dyeing liquid in the process of microwave dyeing fixation, microwave conventional solid color, conventional dyeing microwave solid color at the same time respectively, dilute sample with distilled water to $200 \mathrm{ml}$, and according to D.1 way get dyeing rate.

E.Color fastness test

1.Rubbing fastness test

Use grey color card for color fastness, color fastness rating evaluation [4-5], and according to GB/T 3921-1997"Textile Color Fastness Test,Fastness to washing", GB/T 3920-1997 "Textile Color Fastness Test, Rubbing Fastness", test rubbing fastness tests and soaping fastness test.

\section{II.Results and discussion}

\section{A. Dyeing rate}

1.Conventional solid color dyeing rate

In the case of conventional solid color dyeing process, dyeing ratesof active turquoise blue $\mathrm{KN}-\mathrm{G}$, reactive orange K-R are shown in Figure 2.

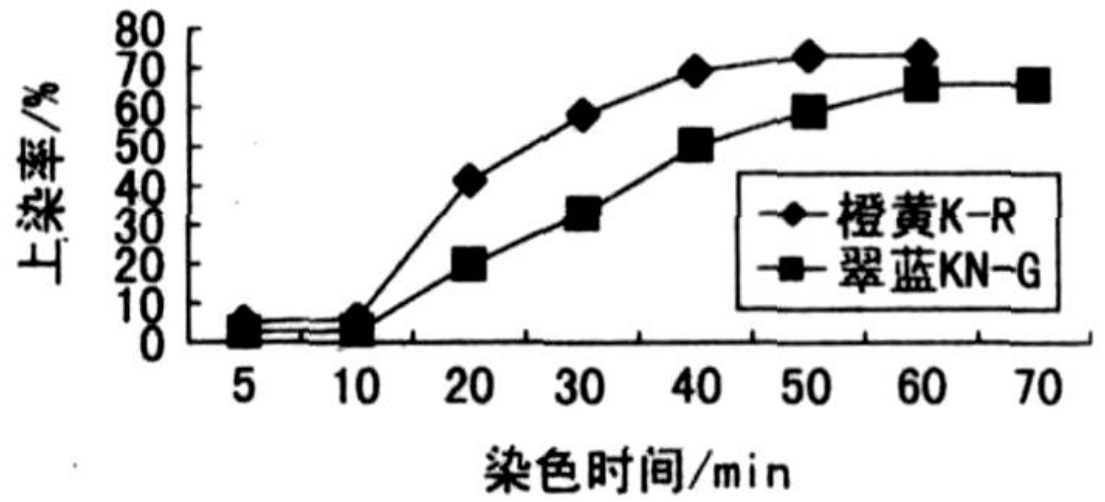

Figure 2 Conventional dyeing curve of solid color dyeing rate 
Figure 2 shows that in the case of conventional solid color dyeing process, the longer dyeing fixation time, the higher dyeing ratesof active turquoise blue $\mathrm{KN}-\mathrm{G}$, reactive orange $\mathrm{K}-\mathrm{R}$ on pure cotton fabric; when time is more than $60 \mathrm{~min}$, the former dyeing rate can reach more than $65.00 \%$, the latter dyeing rate can reach more than $70.00 \%$.

2.Microwave processing dyeing rate

Microwave solid color $4 \mathrm{~min}$, dyeing rates change of active turquoise blue KN-G, reactive orange K-R within 1-6 min is shown in Figure 3.

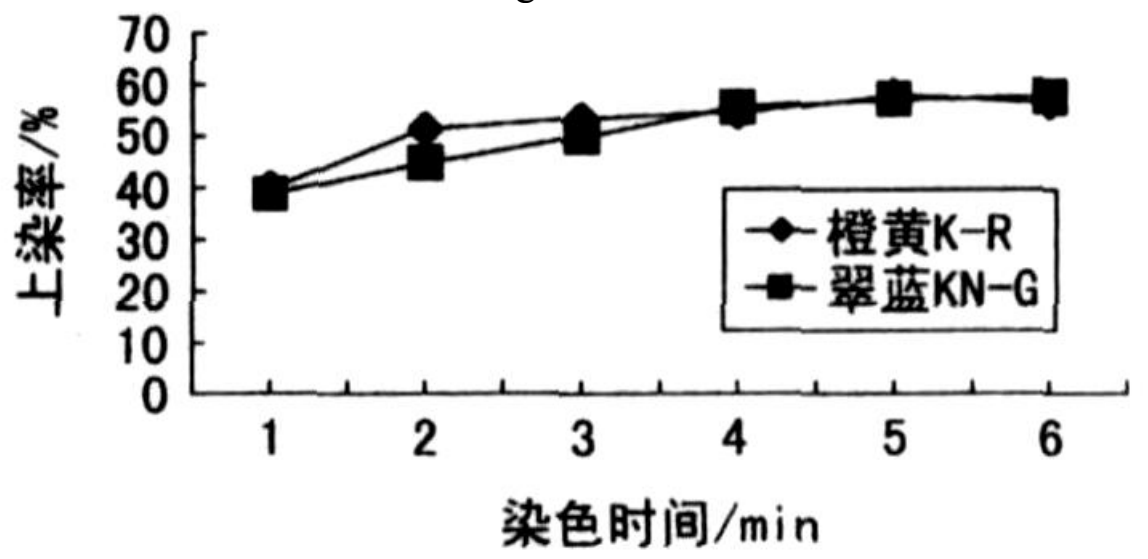

Figure 3 Microwave solid color dyeing rate

Figure 3 shows that the longer microwave dyeing time, the higher dyeing rates of active turquoise blue $\mathrm{KN}-\mathrm{G}$, reactive orange K-R on pure cotton fabric, and when the time is 6 min, both reach the highest dyeing rate, around $57.00 \%$.

We can find that, compared with conventional dyeing fixation, reactive dyes make use of microwave dyeing solid color to achieve the highest dyeing rate with shorter time, but highest dyeing rate is low.

3.Microwave dyeing conventional solid color dyeing rate

Conventional solid color is $30 \mathrm{~min}$, dyeing rates change of active turquoise blue $\mathrm{KN}-\mathrm{G}$, reactive orange K-R by microwave dyeing within 1-6 min is shown in Figure 4.

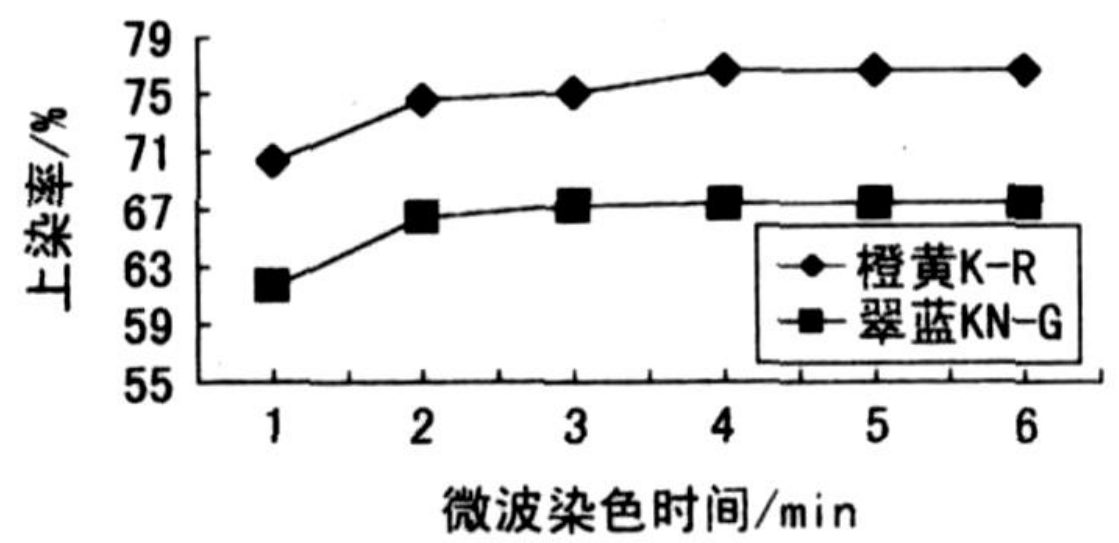

Figure 4 Microwave conventional solid color dyeing rate curve

Figure 4 shows that the longer the microwave dyeing time, the higher dyeing rates of active turquoise blue $\mathrm{KN}-\mathrm{G}$, reactive orange $\mathrm{K}-\mathrm{R}$ on pure cotton fabric, and when the time is 6 min, both reach the highest dyeing rate, around $67.00 \%$ and 76.00 respectively.

We can find that, microwave dyeing and conventional solid colorcan not only shorten dyeing time, also can effectively improve dyeing rate with advantages of conventional high dyeing rate and short microwave dyeing time.

4.Conventional dyeing microwave solid color dyeing rate

Microwave fixation is 4-5 min, dyeing rates change of active turquoise blue $\mathrm{KN}-\mathrm{G}$, reactive orange K-R by conventional dyeing is shown in Figure 5 . 


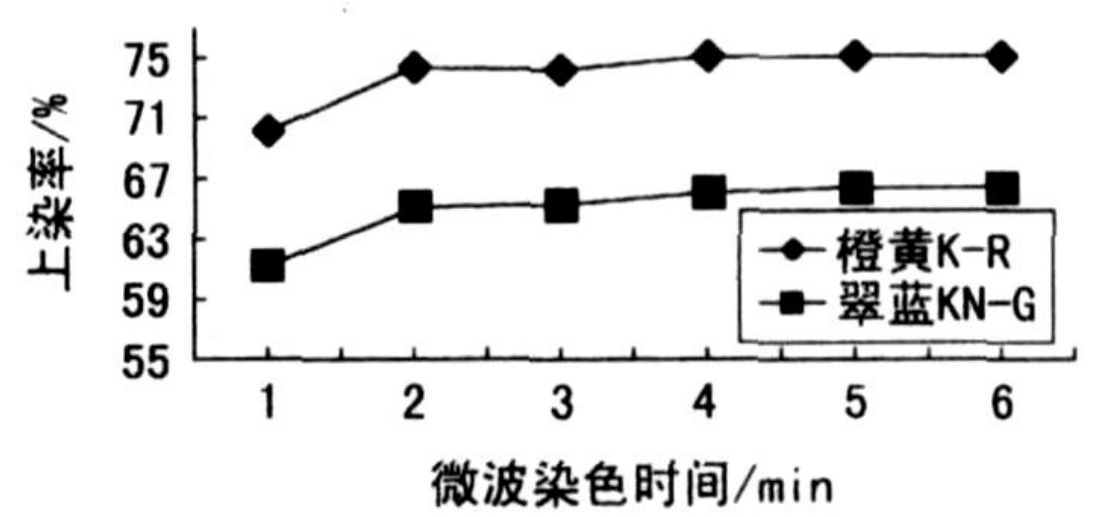

Figure 5 Normal dyeing microwave fixation dyeing rate curves

Figure 5 shows that the longer the conventional dyeing time, the higher dyeing rates of active turquoise blue KN-G, reactive orange K-R on pure cotton fabric, and when the time is 6 min, both reach the highest dyeing rate, around $66.00 \%$ and $75.00 \%$.

We can find that, conventional dyeing and microwave solid color can not only shorten fixation time, also can effectively improve dyeing rate with conventional high dyeing rate and short microwave fixation time.

B.Color fastness

Make Table 1 according to detected reactive dye fastness data.

\begin{tabular}{|c|c|c|c|c|c|}
\hline \multirow[b]{2}{*}{ Dye } & \multirow[b]{2}{*}{ Process } & \multicolumn{2}{|c|}{$\begin{array}{c}\text { Soaping } \\
\text { fastness/level }\end{array}$} & \multicolumn{2}{|c|}{ Crocking/level } \\
\hline & & Color stick & $\begin{array}{c}\text { Color } \\
\text { chang } \\
\mathrm{e} \\
\end{array}$ & $\begin{array}{l}\text { dry } \\
\text { rubbin } \\
\mathrm{g} \\
\end{array}$ & $\begin{array}{c}\text { wet } \\
\text { rubbin } \\
\mathrm{g}\end{array}$ \\
\hline \multirow{4}{*}{$\begin{array}{c}\text { Reactive } \\
\text { turquoise blue } \\
\text { KN-G }\end{array}$} & $\begin{array}{c}\text { Conventional dyeing } \\
\text { solid color }\end{array}$ & 4 & 4 & $4-5$ & 3 \\
\hline & $\begin{array}{l}\text { Conventional dyeing } \\
\text { microwave solid color }\end{array}$ & 4 & 4 & $4-5$ & $4-5$ \\
\hline & $\begin{array}{c}\text { Microwave dyeing solid } \\
\text { color }\end{array}$ & 4 & 4 & $4-5$ & $4-5$ \\
\hline & $\begin{array}{l}\text { Microwave dyeing } \\
\text { conventional solid color }\end{array}$ & 4 & 4 & $4-5$ & 4 \\
\hline \multirow{4}{*}{$\begin{array}{c}\text { Active orange } \\
\text { K-R }\end{array}$} & $\begin{array}{c}\text { Conventional dyeing } \\
\text { fixation }\end{array}$ & $4-5$ & $4-5$ & 5 & 4 \\
\hline & $\begin{array}{l}\text { Conventional dyeing } \\
\text { microwave fixation }\end{array}$ & $4-5$ & $4-5$ & 5 & $4-5$ \\
\hline & $\begin{array}{l}\text { Microwave dyed solid } \\
\text { color }\end{array}$ & $4-5$ & $4-5$ & 5 & $4-5$ \\
\hline & $\begin{array}{c}\text { Conventional microwave } \\
\text { dyeing fixation }\end{array}$ & $4-5$ & $4-5$ & 5 & $4-5$ \\
\hline
\end{tabular}

Table 1 Reactive dyes' dyeing fastness for cotton fabrics

Table 1 shows that compared with conventional dyeing fixation, the soaping fastness and rubbing fastness of reactive turquoise blue $\mathrm{KN}-\mathrm{G}$ or active orange $\mathrm{K}-\mathrm{R}$ have higher technological level in normal dyeing microwave fixation, microwave fixation, microwave conventional solid color dyeing process.

\section{III.Conclusions and recommendations} time.

Conclusion: microwave heating can improve reactive dyes dyeing rate while shortening dyeing 
Advice: for cotton fabrics' dyeing solid color, integrate the use of conventional technology and microwave technology, such as microwave dyeing conventional solid color can improve the dyeing rate of microwave dyeing, and reduce dyeing time; conventional dyeing microwave fixation can shorten fixation time and guarantee higher dyeing rate.

\section{References}

[1] Yang Yingxian, Jiang Yikuan. Application and prospect of microwave technology in dyeing and finishing industry [J]. Journal of Dyeing and Finishing Technology, 2005 (1) : 6-8.

[2]Warren S Perkins, et a.1 Fixation ofReactive DyesUsing Radio-frequency energy[J]. Journalof the Society ofDyers and Colourists, 1992(2): 70-73.

[3]CRISTINA L, TIMOTHY J M. Mi-crowave and ultrasonic processing: Now a realistic option for industry[J].Chemical Engineering and Processing: Process Intensification,2010 (49): 885-900.

[4] FuJingli, Wei Jinhui. Microwave sintering technology application in test production of vanadium nitride [J]. Chengde-Steel Technology, 2006 (1) : 20-21

[5] Zhang Xianru, Xu Zheng. Principle and application of microwave technology in material chemistry progress [J]. Journal of Radiation Research and Radiation Technology, 2005, 23 (4) : 196-200. 